\title{
Two photon versus one photon fluorescence excitation in whispering gallery mode microresonators.
}

\author{
Carme Pastells ${ }^{1,2}$, M.-Pilar Marco ${ }^{1,2}$, David Merino ${ }^{3}$, Pablo Loza-Alvarez ${ }^{3}$, Laura Pasquardini ${ }^{4}$, Lorenzo \\ Lunelli $^{4,5}$ Cecilia Pederzolli ${ }^{4}$, Nicola Daldosso ${ }^{6}$, Daniele Farnesi ${ }^{7,8}$, Simone Berneschi ${ }^{7}$, Giancarlo C. \\ Righini $^{7,8}$, F. Querciolii ${ }^{9}$, Gualtiero Nunzi Conti ${ }^{7}$, Silvia Soria ${ }^{7 *}$ \\ ${ }^{1}$ Nanobiotechnology for Diagnostics group (Nb4Dg), IQAC-CSIC, 08034-Barcelona, Spain \\ ${ }^{2}$ CIBER de Bioingeniería, Biomateriales y Nanomedicina, 08034-Barcelona, Spain \\ ${ }^{3}$ ICFO-Institut de Ciències Fotòniques, 08860-Castelldefels, Barcelona, Spain \\ ${ }^{4}$ Fondazione Bruno Kessler, 38123 Povo (TN), Italy \\ ${ }^{5}$ IBF-CNR, 38123 Povo (TN), Italy \\ ${ }^{5}$ Department of Computer Science, University of Verona, Strada le Grazie 15, 37134 Verona, Italy \\ ${ }^{6}$ CNR-IFAC "Nello Carrara" Institute of Applied Physics, 50019 Sesto Fiorentino (FI), Italy \\ ${ }^{7}$ Museo Storico della Fisica e Centro Studi e Ricerche "E. Fermi”, 00184 Roma, Italy. \\ ${ }^{8}$ CNR-INO National Institute of Optics, Sesto Fiorentino (Fi) Italy \\ *s.soria@ifac.cnr.it
}

\begin{abstract}
We investigate the feasibility of both one photon and two photon fluorescence excitation using whispering gallery mode microresonators. We report the linear and non linear fluorescence real-time detection of labeled IgG covalently bonded to the surface of a silica whispering gallery mode resonator (WGMR). The immunoreagents have been immobilized onto the surface of the WGMR sensor after being activated with an epoxy silane and an orienting layer. The developed immunosensor presents great potential as a robust sensing device for fast and early detection of immunoreactions. We also investigate the potential of microbubbles as nonlinear enhancement platform. The dyes used in these studies are dylight800, tetramethyl rhodamine isothiocyanate, rhodamine $6 \mathrm{G}$ and fluorescein. All measurements were performed in a modified confocal microscope.
\end{abstract}

Keywords: whispering gallery modes resonators, microspheres, non- linear fluorescence 


\title{
Two photon versus one photon fluorescence excitation in whispering gallery mode microresonators.
}

\author{
Carme Pastells ${ }^{1,2}$, M.-Pilar Marco ${ }^{1,2}$, David Merino ${ }^{3}$, Pablo Loza-Alvarez ${ }^{3}$, Laura Pasquardini ${ }^{4}$, Lorenzo \\ Lunelli $^{4,5}$ Cecilia Pederzolli $^{4}$, Nicola Daldosso ${ }^{6}$, Daniele Farnesi ${ }^{7,8}$, Simone Berneschi ${ }^{7}$, Giancarlo C. \\ Righini $^{7,8}$, F. Quercioli ${ }^{9}$, Gualtiero Nunzi Conti ${ }^{7}$, Silvia Soria ${ }^{7 *}$ \\ ${ }^{1}$ Nanobiotechnology for Diagnostics group (Nb4Dg), IQAC-CSIC, 08034-Barcelona, Spain \\ ${ }^{2}$ CIBER de Bioingeniería, Biomateriales y Nanomedicina, 08034-Barcelona, Spain \\ ${ }^{3}$ ICFO-Institut de Ciències Fotòniques, 08860-Castelldefels, Barcelona, Spain \\ ${ }^{4}$ Fondazione Bruno Kessler, 38123 Povo (TN), Italy \\ ${ }^{5}$ IBF-CNR, 38123 Povo (TN), Italy \\ ${ }^{5}$ Department of Computer Science, University of Verona, Strada le Grazie 15, 37134 Verona, Italy \\ ${ }^{6}$ CNR-IFAC "Nello Carrara" Institute of Applied Physics, 50019 Sesto Fiorentino (FI), Italy \\ ${ }^{7}$ Museo Storico della Fisica e Centro Studi e Ricerche "E. Fermi”, 00184 Roma, Italy. \\ ${ }^{8}$ CNR-INO National Institute of Optics, Sesto Fiorentino (Fi) Italy \\ *s.soria@ifac.cnr.it
}

\begin{abstract}
We investigate the feasibility of both one photon and two photon fluorescence excitation using whispering gallery mode microresonators. We report the linear and non linear fluorescence real-time detection of labeled IgG covalently bonded to the surface of a silica whispering gallery mode resonator (WGMR). The immunoreagents have been immobilized onto the surface of the WGMR sensor after being activated with an epoxy silane and an orienting layer. The developed immunosensor presents great potential as a robust sensing device for fast and early detection of immunoreactions. We also investigate the potential of microbubbles as nonlinear enhancement platform. The dyes used in these studies are dylight800, tetramethyl rhodamine isothiocyanate, rhodamine $6 \mathrm{G}$ and fluorescein. All measurements were performed in a modified confocal microscope.
\end{abstract}

Keywords: whispering gallery modes resonators, microspheres, non- linear fluorescence

\section{INTRODUCTION}

Whispering gallery mode resonators (WGMR) are evanescent wave sensors that can be used both as refractometres or label-free sensors and fluorescence based sensors [1, 2]. The disadvantages of the labeled system - namely, cost and possibly reduced reactivity - are normally compensated by lower limit of detection (LOD), while in the case of direct monitoring, the limitations lie in the ineffectiveness of detecting small 
molecular weight analytes and in the sensitivity to non-specific binding. In a WGMR light is trapped by total internal reflection at the resonator interface and the evanescent tail of the electromagnetic field interacts with the analytes on the resonator surface. WGMR can achieve very high quality factors $Q$ [3] which means high sensitivity. A crucial step for producing reliable biosensors is the surface functionalization, or chemical modification of the transducer surface in order to bind the biological recognition element on it. This functional layer has to be very thin, between 10-100 nm and homogeneous, in order to preserve the high quality of the transducer and the interaction with the sensing layer and the WGMR $[4,5]$. The permanence of high $Q$ values after the functionalization of the surface is an essential requirement in order to achieve highly sensitive devices.

For that reason, most of the efforts have been directed to label-free detection, even though the feasibility of using WGMR as platforms for one photon fluorescence (OPF) detection has been studied [6]. Based on these recent papers, we tested first the feasibility of OPF with spherical WGMR where antibodies against Staphyolococcus aureus cell wall labeled with a near infrared dye (Dylight800, Thermo Scientific) were immobilized on its surface. S. aureus is a bacterium that can cause a range of illnesses, from minor skin infections to life threatening diseases. Moreover, this pathogen is one of the five most common causes of nosocomial infections.

It is true that commercial NIR dyes are excellent but their intrinsically small Stokes shift may produce excitation and scattered light interferences, especially when the excitation light used is a tunable diode laser. The wide gain profile of the semiconductor easily masks the emitted fluorescence due to the overlap with the emission spectrum of the dye [7]. Being that our case, we decided to resort to a nonlinear detection technique like two photon fluorescence (TPF), which was already validated as a very good detection technique for labeled peptides [8] and steroids $[9,10]$

As mentioned above, TPF measurements with near infra-red radiation have a number of advantages over measurements with OPF. Specifically, the large energy gap between the excitation and emission radiation reduces the background noise, the static photobleaching of the dyes that are used is reduced because there is a quadratic dependence of the absorption on intensity [11,12], and the use of near infra-red radiation (NIR) minimizes the photodamage of cells and tissue thereby lowering autofluorescence. On the other hand, conventional TPF requires highly intense and focused laser light of instantaneous photon flux densities of at least $10^{31}$ photons $/ \mathrm{cm}^{2}$, which in turn lowers the photodamage threshold [13]. In order to overcome such a difficulty, i.e. to achieve the needed energies and yet avoid tight focusing, we resort to low-loss, high-quality factor whispering gallery mode resonators (WGMR) [1,14]. Another reason to use TPF is the available wide range of visible fluorescent dyes with very high quantum yields and molar extinction coefficients.

The TPF measurements were performed in a modified confocal microscope; we coupled the excitation light with a $4 \mathrm{X}$ and 10X objective and detected the TPF signal with a CCD camera. We performed all measurements with a femtosecond laser (Ti:Sapphire, Coherent) in order to avoid secondary effects in the biological layers or in the organic solutions. We report the observation of TPF, first in microbubbles WGMR filled with a $10^{-3} \mathrm{M}$ and $10^{-4} \mathrm{M}$ solution of fluorescein and then $10^{-6} \mathrm{M}$ solution of Rhodamine $6 \mathrm{G}$, in the latter the concentration is three orders of magnitude lower than previous work [14]; and in microspheres coated with labeled IgG.

\section{EXPERIMENTAL RESULTS AND DISCUSSION.}

\subsection{Surface functionalization Method.}


The microspheres were functionalized following three different procedures. The first batch of microspheres were functionalized as follows: before exposing to the epoxy silanes, microspheres were soaked into piranha solution $\left(\mathrm{H}_{2} \mathrm{SO}_{4}: \mathrm{H}_{2} \mathrm{O}(7: 3)\right)$ for 3 min minutes to remove organic contaminants and oxidize the surface, then rinsed in MilliQ water, soaked in $10 \% \mathrm{NaOH}(\mathrm{w} / \mathrm{v})$ for $1 \mathrm{~h}$, washed again in milliQ water and absolute EtOH. The activated spheres were then soaked in 98\% (3-glycidyloxypropyl)-trimethoxysilane (GPTMS) for 1h, dried in air and stored under vacuum. The epoxy-derivatized microspheres were coated with PSau2-BSA $(0.125 \mu \mathrm{g} / \mathrm{mL}$ in printing buffer $-150 \mathrm{mM}$ sodium phosphate buffer, $0.001 \%$ sodium dodecyl sulfate, $\mathrm{pH} 8.5)$ $3 \mathrm{~h}$ at room temperature (RT). Then, the microspheres were washed with PBST (10 mM phosphate buffer on a $140 \mathrm{mM} \mathrm{NaCl}$ solution with $0.05 \%$ Tween $20, \mathrm{pH} 7.5$ ) and the specific antibody (As $204,0.02 \mathrm{mg} / \mathrm{mL}$ in PBST) was added for $30 \mathrm{~min}$ at RT. After another cycle of wash with PBST, the microspheres were labelled with commercially anti-IgG conjugated to tetramethyl rhodamine isothiocyanate (TRITC) $(0.02 \mathrm{mg} / \mathrm{mL}$ in PBST). Then, the microspheres were washed with PBST and MilliQ water and the fluorescent derivatization of the microspheres was verified. The uniformity and fluorescence emission of the IgG-TRITC was checked in a commercial confocal microscope (OPF) (figure 1.a)

The second batch of microspheres were functionalized and coated with PSau2-BSA following the same procedure described for the first batch. Then, the microspheres were washed with PBST and the specific antibody labeled with the dylight 800 fluorophore (As204-dylight800, $0.02 \mathrm{mg} / \mathrm{mL}$ in printing buffer) was added for $30 \mathrm{~min}$ at RT. The As204-dylight800 conjugate was prepared in one of our labs, three different molar ratios of As204:dylight800 were tested: 1:1;1:2 and 1:10). After, the microspheres where washed with PBST and finally with MilliQ water. In order to verify the fluorescent derivatization of the microspheres, we checked the uniformity and fluorescence emission of the As204-dylight800 with a LICOR Odyssey scanner Figure 1.b. shows the ratio 1:2 as an example, since all molar ratios were uniform and gave good signal.

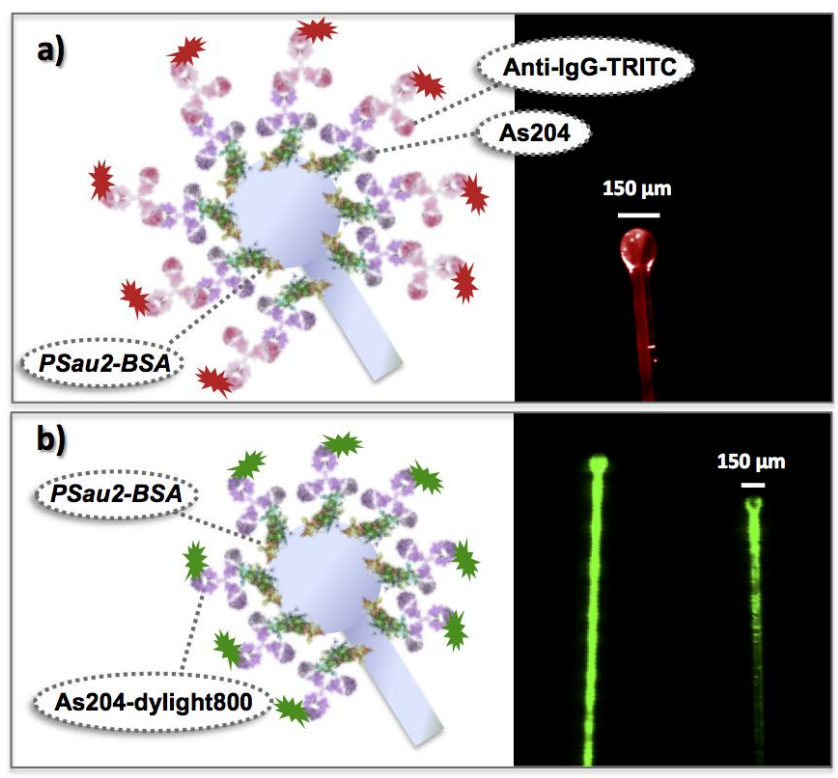

Fig. 1. a) Microsphere labelled with Anti-IgG-TRITC (fluorescence 2.5X) and b) microsphere labelled with As204dylight800 (molar ratio 1:2). 
The third batch of microspheres was functionalized as follows: after oxidizing the surface with the piranha solution, $\left(\mathrm{H}_{2} \mathrm{SO}_{4}: \mathrm{H}_{2} \mathrm{O}_{2}(4: 1 \mathrm{v} / \mathrm{v})\right.$ for $\left.3 \mathrm{~min}\right)$, the spheres were immersed in a $0,01 \%$ solution of 3 glycidoxypropyl-trimethoxysilane (GPTMS) in anhydrous toluene at $60^{\circ} \mathrm{C}$ for $1 \mathrm{~min}$. On this second batch, in order to avoid random orientation of the antibodies we immobilized covalently an orienting layer of protein $\mathrm{G}$ (10 $\mathrm{mg} / \mathrm{ml}$ in phosphate buffer $100 \mathrm{mM} \mathrm{pH} 8$ for 2 hours) that binds antibodies with a high affinity through the Fc region, leaving the Fab sites free for interaction. Then we bound: a) As204 conjugated with dylight 800 (1:2 and 1:10) and b) IgG labelled with tetramethyl rhodamine isothiocyanate (TRITC) both at $8 \mu \mathrm{g} / \mu \mathrm{lml}$ in phosphate buffer $100 \mathrm{mM} \mathrm{pH} 8$ at room temperature for two hours.

Dylight 800 is a fluorescent dye with a maximum absorption at $777 \mathrm{~nm}$ and a maximum emission at $794 \mathrm{~nm}$ (Pierce, datasheet). However, the physical characteristics of fluorescent dyes change depending on the surroundings (emission and excitation wavelengths and also lifetime). This is especially true when dyes are covalently bound to biomolecules and dried monolayers. We checked the fluorescence emission of a solution of As204-dylight800 (molar ratio 1:10) in phosphate buffer (100 mM, pH 8) for four different excitation wavelengths $\left(770,772,775\right.$ and $777 \mathrm{~nm}$ ) using a FluoroMax ${ }^{\circledR}-4$ Fluorescence Spectrophotometer (HORIBA Scientific). As it can be seen in figure 2.a., the maximum emission occurs at $788 \mathrm{~nm}$ independently on the excitation wavelength (between 770 and $777 \mathrm{~nm}$ ) whereas the maximum excitation wavelength is $770 \mathrm{~nm}$. Figure 2.b. shows the emission spectra of a mock microsphere (silica glass slide functionalised with and without a covalently bound layer of As204-dylight800), analyzed by using the Fluorescence Spectrometer. The maximum emission is shifted towards longer wavelength of about $13 \mathrm{~nm}$ with respect to the dylight800 conjugated As204 solution, as expected [7].
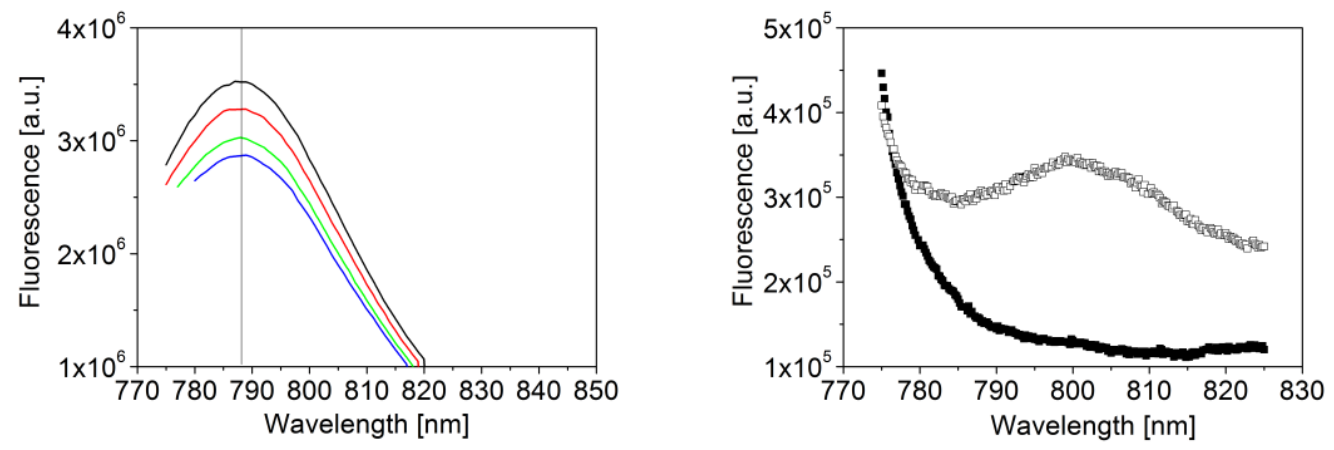

Fig. 2. a) Emission spectra of a dylight800 conjugated As204 solution for different excitation wavelengths (black: 770nm; red: $772 \mathrm{~nm}$; green: $775 \mathrm{~nm}$ and blue: $777 \mathrm{~nm}$ ); b) Emission spectra of a mock microsphere in two different conditions: empty squares: silica glass slide mimicking the 3rd batch of microspheres; filled squares: functionalized silica glass slide without the bioconjugate As204-dylight800.

\subsection{One photon fluorescence measurements.}


The experimental setup for measuring the quality factor and OPF is sketched in Fig.3. The light from a fiber pigtailed tunable diode laser (TDL) is tunable from $765 \mathrm{~nm}$ to $781 \mathrm{~nm}$. A polarization controller and a tap coupler (5\%, not shown in Fig. 3) allow adjusting the polarization state and monitoring the lunched pump power. Light is then coupled to the WGMR by means of a fiber taper, also produced in-house. The laser is tuned into a resonance from high to low frequencies, which results in thermal self-locking [15] of the WGMR mode to the pump laser. Fluorescence was detected on an optical spectrum analyzer (OSA) or a spectrometer by collecting with a multimode fiber (MMF, $50 \mu \mathrm{m}$ core, $0.2 \mathrm{NA}$ ) the light scattered from the microsphere using a long pass filter (RG780, Schott). A $3 \mathrm{~dB}$ splitter at the fiber output (not shown in Fig. 3) has one end on the spectrometer and the other on a detector connected to an oscilloscope, which allows locating the resonance positions while scanning the laser.

Silica microspheres can be easily fabricated directly on the tip of a standard telecom fiber using a sequence of arc discharges of a fiber fusion splicer [16]. We fabricated spheres of different diameters, ranging from about $125 \mu \mathrm{m}$ to $180 \mu \mathrm{m}$. The residual fiber stem is then mounted on a translation stage with piezoelectric actuators and a positioning resolution of $20 \mathrm{~nm}$.

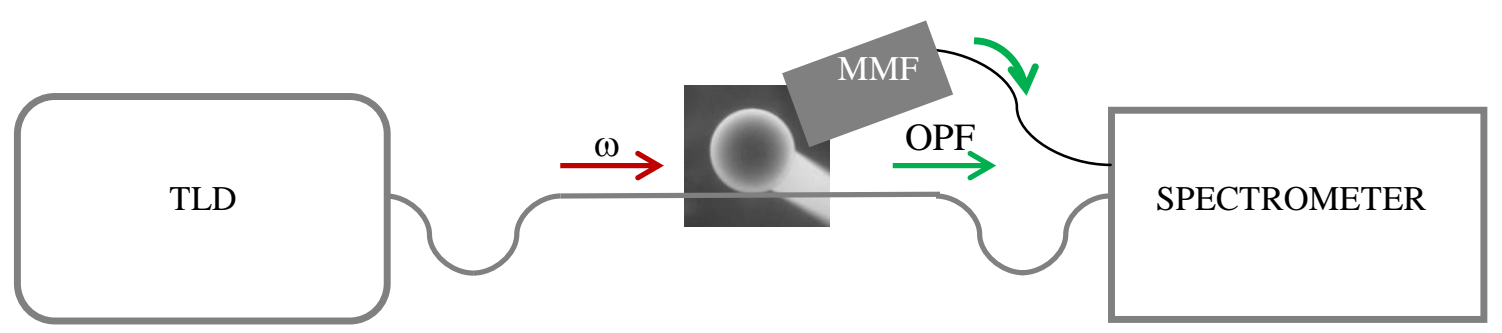

Fig. 3. Scheme of the experimental setup. TLD: Tunable laser diode; MMF: multimode fiber, OPF: one photon fluorescence.

We measured the quality factor of the functionalized microspheres at two wavelengths: at $1550 \mathrm{~nm}$ (without absorption) and at $770 \mathrm{~nm}$ (maximum absorption). The quality factor at $1550 \mathrm{~nm}$ of the first and second batch of microspheres were in excess of $10^{6}$ whereas for the third one the quality factor was in excess of $10^{7}$ (not shown in here). At $770 \mathrm{~nm}$, due to the absorption of the dye, the Q factor lowered by one order of magnitude for both functionalized microspheres (figure 4).
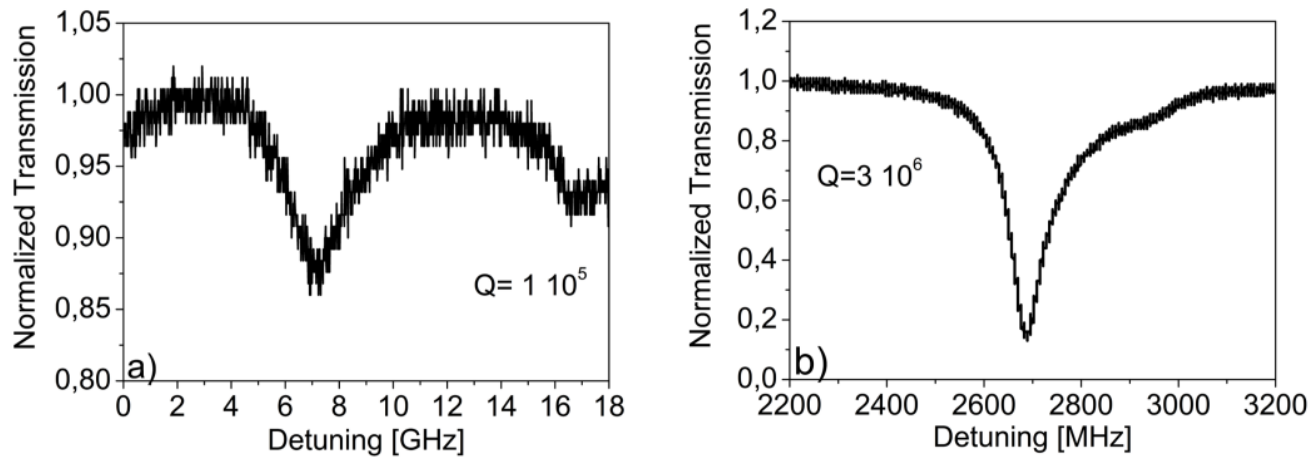
Figure 4. Q factor at $770 \mathrm{~nm}$ of the microspheres with 1:2 ratio As204-dylight800 covalently bound to the surface: a) first batch and b) third batch.

In principle, the evanescent tail of the WGM should be overlapping with the biological layer and interacting with the As204-Dylight800. With this idea in mind, we proceeded to measure the scattered OPF with a MMF connected to a hand held spectrometer. Figure 5.a. and 5.b. show the collected scattered light for a microsphere of at diameter of about $180 \mathrm{um}$ and the laser emission spectrum, respectively. In figure 5.a the excitation wavelength was centered at $770 \mathrm{~nm}$ and the power of the laser was attenuated from $148 \mu \mathrm{W}$ down to $245 \mathrm{nW}$. All microsphere batches show similar behavior.

Despite of how promising the graphs look, there is a main discrepancy between the emitted OPF from the surface of the microsphere and the OPF measured with a commercial scanner: the emission peak was expected at $814 \mathrm{~nm}$ for a dry environment (fig.2.b) and in the graphs it is at $780 \mathrm{~nm}$. The center wavelength of the TLD is at $770 \mathrm{~nm}$ with an output power $(1 \mathrm{~mW})$ but the gain bandwidth of the laser is extended from 760 to $790 \mathrm{~nm}$ and at $780 \mathrm{~nm}$ the power is about $0.1 \mu \mathrm{W}$ with a shape that resembles too much the spectra shown in fig.5.a. without the convolution of the filter (see fig.5.b).
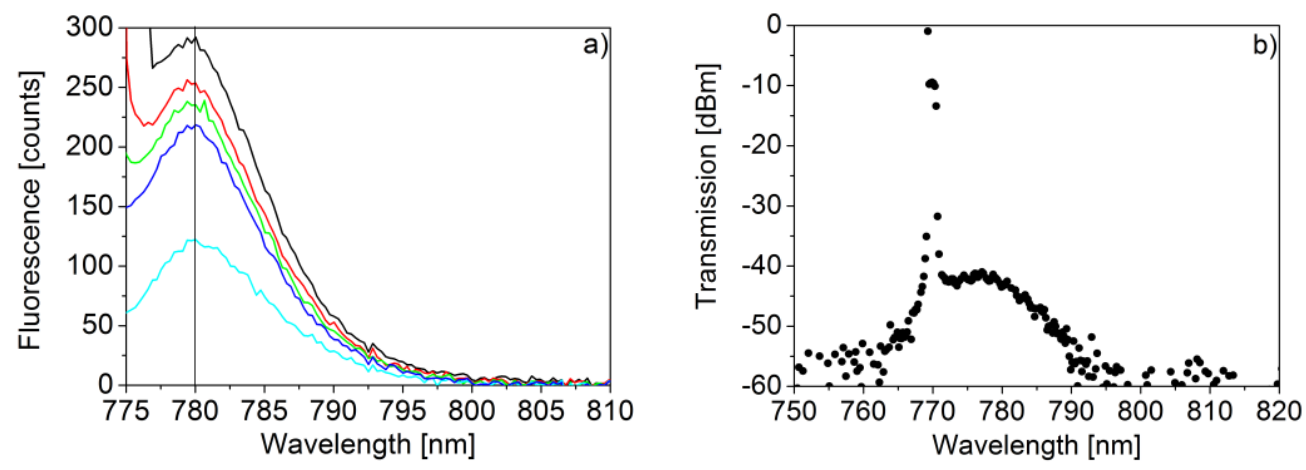

Fig. 5. a) Scattered light measured with a hand held spectrometer from the second batch of microspheres with As204Dylight800 1:2 ratio As204-Dylight800 covalently bound to the surface microsphere of a diameter of about $140 \mu \mathrm{m}$ (black $148 \mathrm{~mW}$, red $92 \mathrm{~mW}$, gree $42 \mathrm{~mW}$, blue $2 \mathrm{~mW}$, cyan $398 \mathrm{nW}$ ); b) Laser spectrum measured with an OSA.

In order to discriminate whether the scatter light we measured is OPF or mainly the scattered excitation laser light, we tuned the excitation wavelength to the red edge of the bandwidth of the laser, namely, $788 \mathrm{~nm}$. At this wavelength, there is no excitation light that can heavily interfere with the OPF of the dye. We didn't observe OPF, not even for larger integration times and more averaged signal. We concluded that the measured scattered signal is just the excitation laser scattered by the microsphere and convoluted by the filter. For that 
reason we resorted to TPE of microspheres covalently bound IgG labeled with TRITC ( $8 \mu \mathrm{g} / \square 1 \mathrm{in} 100 \mathrm{mM}$ phosphate buffer, $\mathrm{pH} 8$ ).

\subsection{Two photon excitation in microbubbles and microspheres.}

The MBRs were fabricated from slightly pressurized silica capillaries using a modified fusion splicer, where the electrodes could rotate by $360^{\circ}$ by means of a step by step motor. Details of the fabrication can be found in our previous work [17]. Reproducibility is ensured by the control of all physical parameters of the process (duration and power of arc discharges, gas pressure, rotation speed, distance between capillary and electrodes). The microbubbles were the filled with a $10^{-3} \mathrm{M}$ and $10^{-4} \mathrm{M}$ solution of fluorescein and $10^{-6} \mathrm{M}$ solution of Rhodamine $6 \mathrm{G}$.

We used a modified confocal microscope for coupling the light into the microbubble resonator [18]. We used an inverted light microscope (Nikon Eclipse TE2000U). It can be used as a bright-field microscope or as a phase contrast microscope. However its extendible design and advanced features make it ideal for upgrading it to a multi-modal imaging system. The objectives that we used for this experiment was a $4 \mathrm{X}$ and $10 \mathrm{X}$ $0.5 \mathrm{NA}$ dry objective. Due to the impressive average power capability, broad tuning range and short pulse duration we used a commercial diode pumped, mode locked Ti: Sapphire laser (Coherent-MIRA 900f) for our microscope. It has an average power of $1.2 \mathrm{~W}$, producing femtosecond pulses $(150 \mathrm{fs})$ at a repetition rate of $76 \mathrm{MHz}$. This instrument is tunable over a wavelength range of $690-950 \mathrm{~nm}$ within which falls the twophoton absorption spectra of many fluorophores [19]. The wavelength in the experiments was set to $800 \mathrm{~nm}$. We set the voltage of the galvanometric mirrors to 0 in order to stop the raster scan of the beam and excite the WGM of the microbubble by focusing the laser beam tangential to the bubble wall. The excitation light was filtered by a dichoric mirror (FF720-SDi01, Semrock) and a BG39 Schott filter. We tested first the bubble with the fluorescein filling that was imaged with a $4 \mathrm{X}$ dry objective using a CCD camera in order to see the complete WGM at the equator. Figure 6.a. shows the TPF band around the equator and the TPF partially coupled back to the MBR wall. The two lobes that correspond to the WGM are clearly seen (fig.6.b)
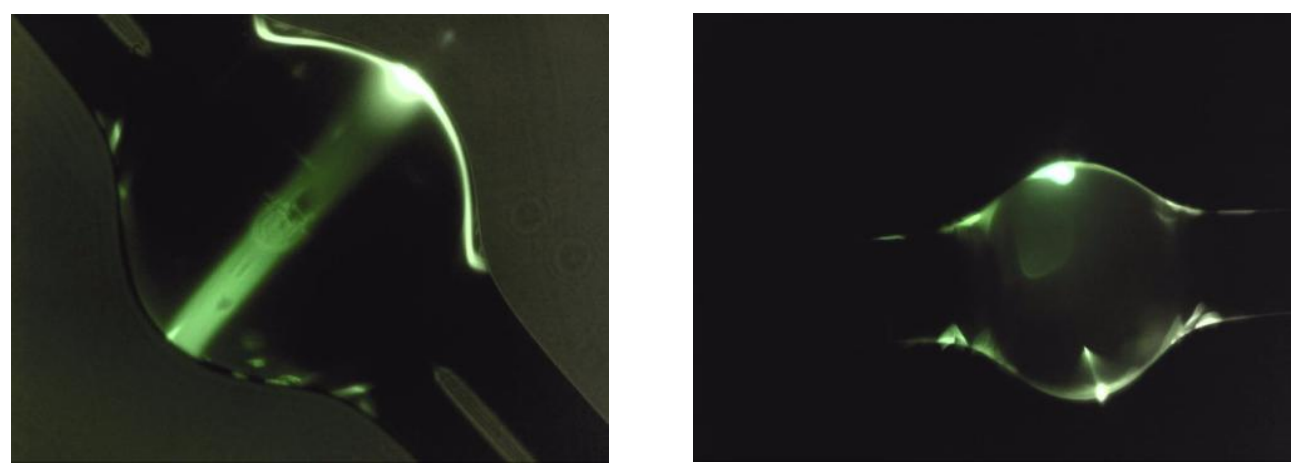

Fig.6. Fluorescence image of two different microbubbles, one filled with $10^{-3} \mathrm{M}$ (a) and $10^{-4} \mathrm{M}$ (b) fluorescein solution, showing the TPF band. The image was taken with a $4 \mathrm{X}$ dry objective. The TPF coupled back to the MBR wall can be also seen. 
The two-photon nature of the emitted signal was validated by checking its dependence on the excitation laser power. Figure 7 shows a logarithmic representation of the TPEF signal from a Rhodamine 6G filled microbubble versus incident laser power at the focal plane. A linear fit to the data has slope $1.8 \pm 0.1$,

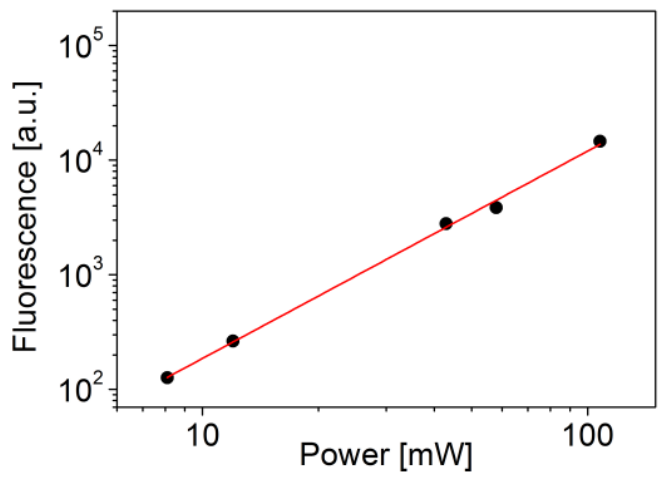

ensuring the quadratic dependence of the obtained signal.

Figure 7. Logarithmic presentation of the TPEF signal from the MBR filled with $10^{-6} \mathrm{M}$ Rhodamine $6 \mathrm{G}$ solution versus incident laser power in log-log scale. The red line is the linear fit with slope close to 2 .

After these results, we tested a microsphere where a layer of IgG labeled with TRITC was bound to its surface. Figure 8 shows the optical image of the microsphere where the coupling point and a partial WGM can be seen.

Figure 8. TPF image of the WGM excited in a microsphere, the coupling point and a partial WGM.

\section{CONCLUSION}

We have demonstrated the potential of microbubbles as nonlinear enhancement platform and verified the feasibility of exciting TPF via WGM in microspheres where labeled IgGs were covalently bound to its surface. The functionalization 
process proved to be uniform, maintaining a good quality factor of the microresonators. In contrast to previous reports of one photon fluorescence, we have demonstrated the impossibility of distinguish OPF from the excitation laser using WGMR.

\section{ACKNOWLEDGEMENTS}

S. Soria acknowledges CNR funding from the STM 2014 program. CNR-IFAC and IQAC-CSIC teams acknowledge funding from the CSIC-CNR bilateral projects. Funding from Centro Fermi is gratefully acknowledged. Funding from Progetto Premiale CNR (2013-2015) is acknowledged. D. Farnesi is a PhD student at the University of Parma. F. Baldini and A. Giannetti are gratefully acknowledged for their support and discussions.

\section{REFERENCES}

[1] S. Soria, S. Berneschi, M. Brenci, F. Cosi, G. Nunzi Conti, S. Pelli and G.C. Righini, Optical microspherical resonators for biomedical applications, Sensors 11 (2011) 785.

[2] F. Vollmer, S. Arnold, Whispering-gallery-mode biosensing: label-free detection down to single molecules, Nat. Methods 5 (2008) 591-596

[3] M. L. Gorodetsky, A. A. Savchenkov, V. S. Ilchenko, Ultimate Q of optical microspherical resonators, Opt. Lett. 21 (1996) 453-455

[4] J. L. Nadeau, V. S. Ilchenko, D. Kossakovski, G. H. Bearman and L. Maleki, High-Q whisperinggallery mode sensor in liquids, Proceedings of SPIE 4629 (2002) 172-180.

[5] S. Soria, F. Baldini, S. Berneschi, F. Cosi, A. Giannetti, G. Nunzi Conti, S. Pelli, G.C. Righini, B. Tiribilli, High-Q polymer-coated microspheres for immunosensing applications. Opt. Express 17 (2009) 14694-14699.

[6] L. M. Freeman, S. Li, Y. Dayani, H-S. Choi, N. Malmstadt, A. M. Armani, Excitation of Cy5 in selfassembled lipid bilayers using optical resonators, Appl. Phys. Lett. 98 (2011)143703

[7] C. Pastells, M. P. Marco, D. Merino, P. Loza-Alvarez, L. Pasquardini, C. Pederzolli, d. Farnesi, S. Berneschi, G. C. Righini, G. Nunzi Conti, S. Soria, Nonlinear fluorescence excitation of Rhodamine 6G and TRITC labeld IgG in whispering gallery mode microresonators, Proc. SPIE 9343 (2015) doi:10.1117/12.2079112

[8] André Selle, Christoph Kappel, Mark Andreas Bader, Gerd Marowsky, Kathrin Winkler, and Ulrike Alexiev, Picosecond pulse induced two photon fluorescence enhancement in biological material by application of grating waveguide structures, Opt. Lett 30 (2005) 1683-1685.

[9] A. Thayil K.N., A. Muriano, P. Salvador, R. Galve, M.P. Marco, D. Zalvidea, P. Loza-Alvarez, T. Katchalski, E. Grinvald, A.A. Friesem, and S. Soria, Nonlinear immunofluorescent assay for androgenic hormones based on grating waveguide structures, Opt. Express, 16 (2008) 13315-13322

[10] A. Muriano, A. Thayil K.N., P. Salvador, R. Galve, P. Loza-Alvarez, S. Soria and M.P. Marco, Twophoton fluorescent immunosensor for androgenic hormones using resonant grating waveguide structures, Sens. Actuators B 174 (2012) 394-401

[11] W. Denk, J.H. Strickler, and W. W. Webb, Two photon laser scanning fluorescence microscopy, Science 248 (1990) 73-76.

[12] P. Schwille, U. Haupts, S. Maiti, and W.W. Webb, Molecular dynamics in living cells observed by fluorescence spectroscopy sith one and two photon excitation, Biophys. J. 77 (1999) 2251-2265.

[13] P.S. Dittrich and P. Schwille, Photobleaching and stabilization of fluorophores used for single molecule analysis with one and two photon excitation, Appl. Phys. B 73 (2001) 829-837

[14] G. A. Cohoon, K. Khieu, and R.A. Norwood, Observation of two photon fluorescence of Rhodamine 6G in microbubble resonators, Opt. Lett. 39 (2014) 3098-3101. 
[15] T. Carmon, L. Yang and K. Vahala, Dynamical thermal behavior and thermal self-stability of microcavities, Opt. Express 12 (2004) 4742-4750

[16] M. Brenci, R. Calzolai, F. Cosi, G. Nunzi Conti, S. Pelli and G. C. Righini, Microspherical resonators for biophotonic sensors, Proceedings of SPIE 6158 (2006). 61580S

[17] S. Berneschi, D. Farnesi, F. Cosi, G. Nunzi Conti, S. Pelli, G.C. Righini, and S. Soria, High Q microbubble resonators fabricated by arc discharge, Opt. Lett. 36 (2011) 3521-3522.

[18] L.L. Martin, P. Haro-Gonzalez, I. R. Martin, D. Navarro-Urrios, D. Alonso, C. Perez-Rodriguez, D. Jaque and N. E. Capuj, Whispering gallery modes in glass microspheres: optimizing of pumping in a modified confocal microscope, Opt. Lett. 36 (2011) 615-617

[19] F. Bestvater, E. Spiess ,G. Stobrawa, M. Hacker, T. Feurer, T. Porwol ,U. Berchner-Pfannschmidt ,C. Wotzlaw and H. Acker, Two-photon fluorescence absorption and emission spectra of dyes relevant for cell imaging, J. Microsc. 208 (2002)108-115 
Click here to download high resolution image
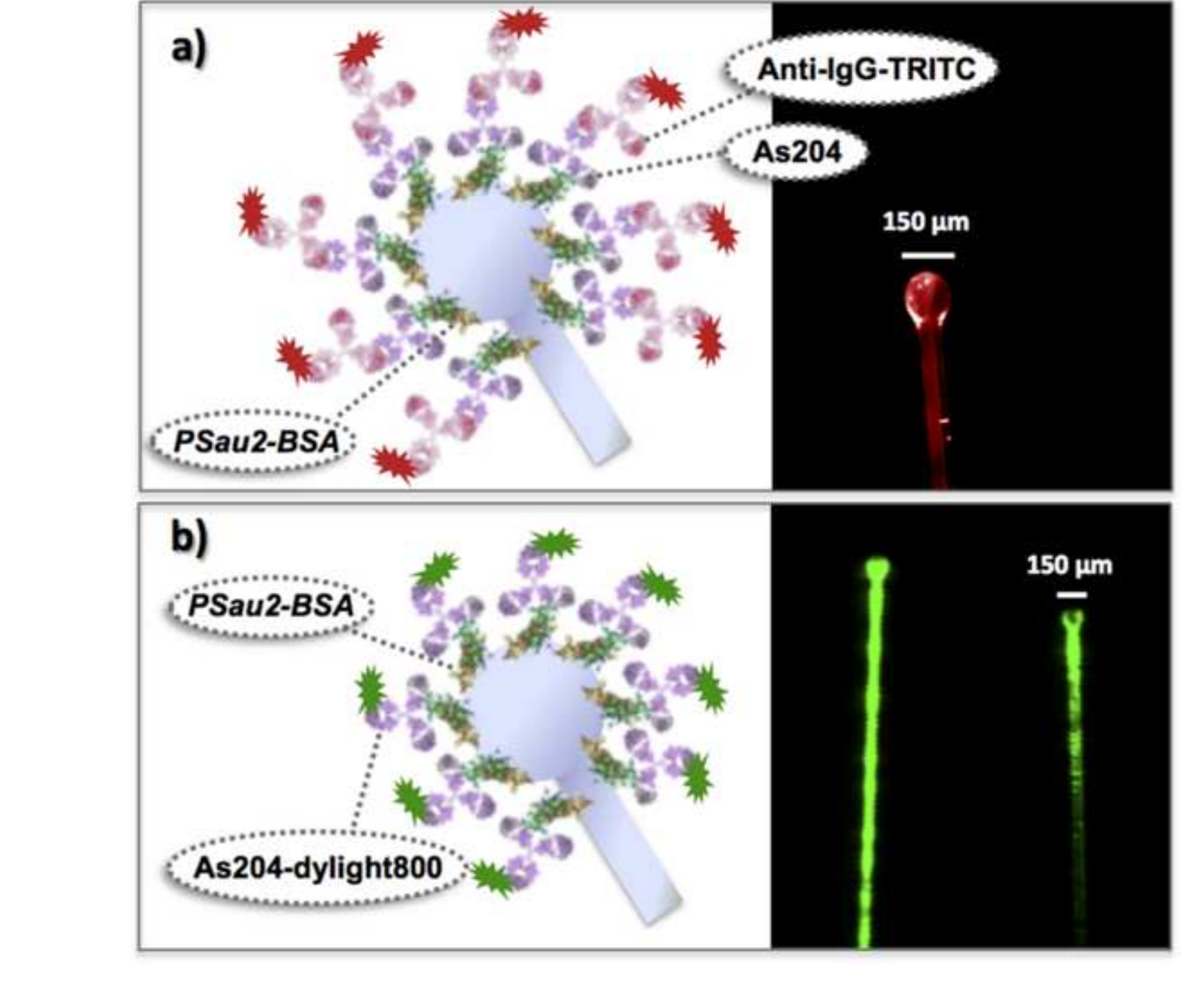


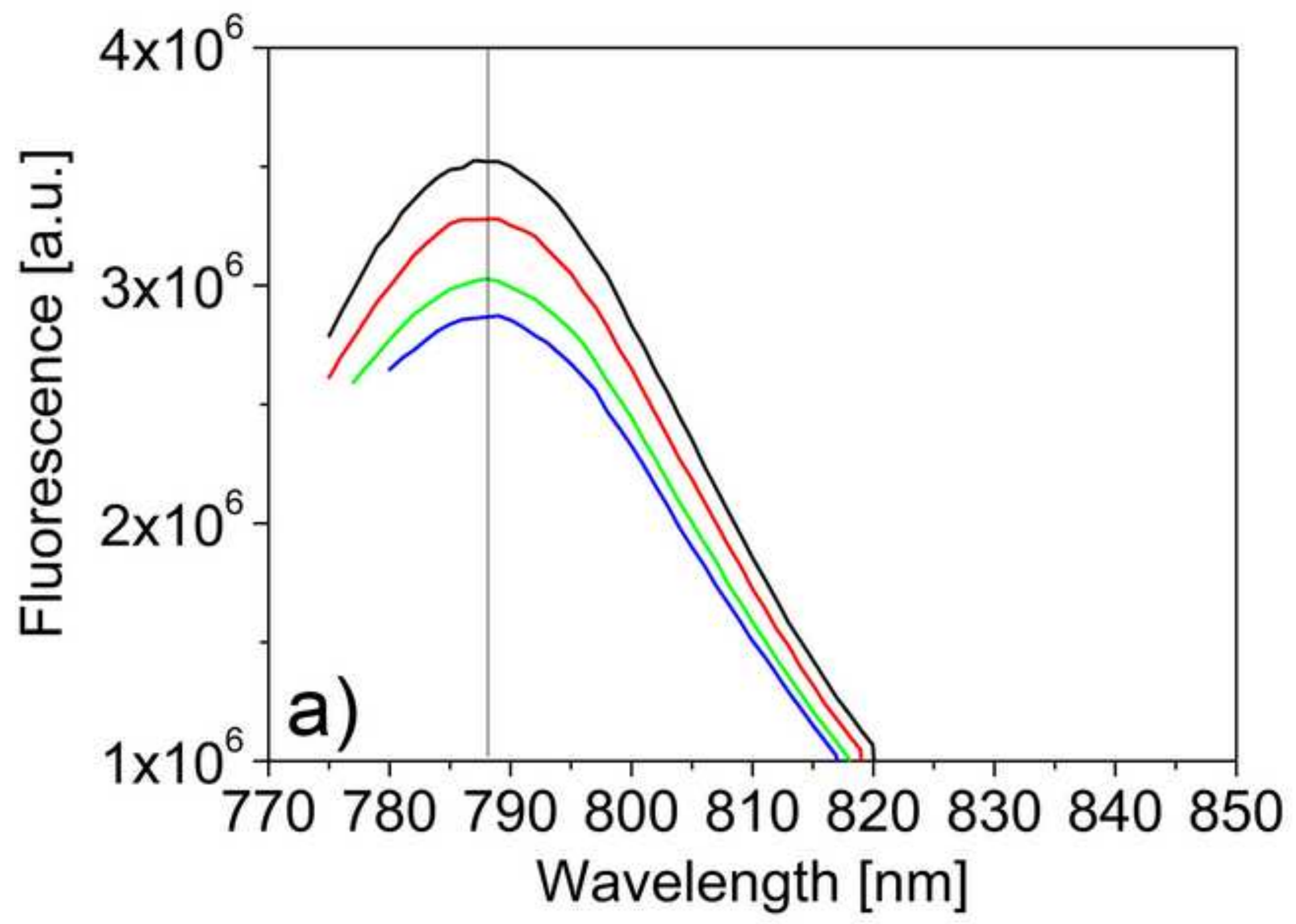




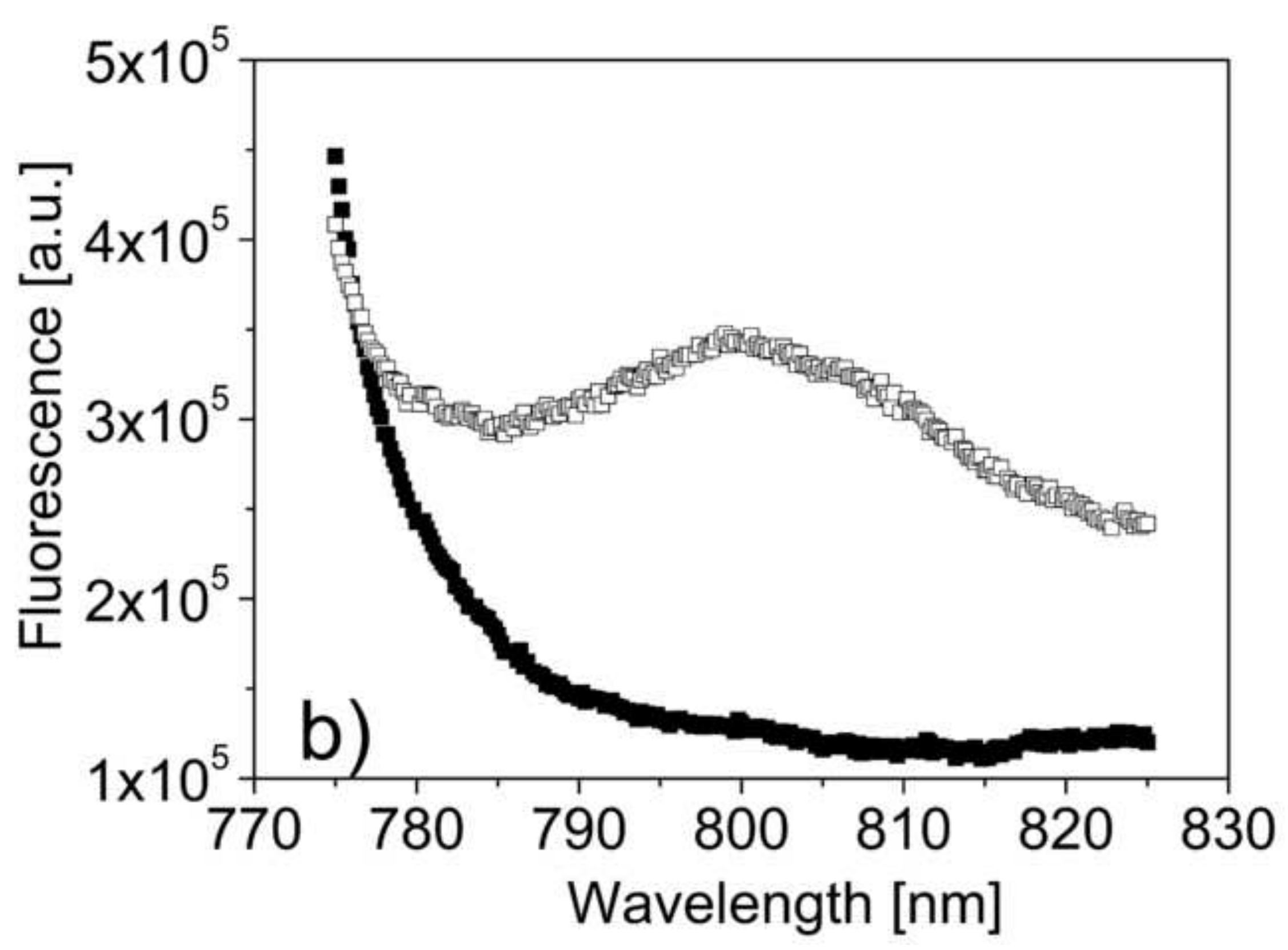


Click here to download high resolution image

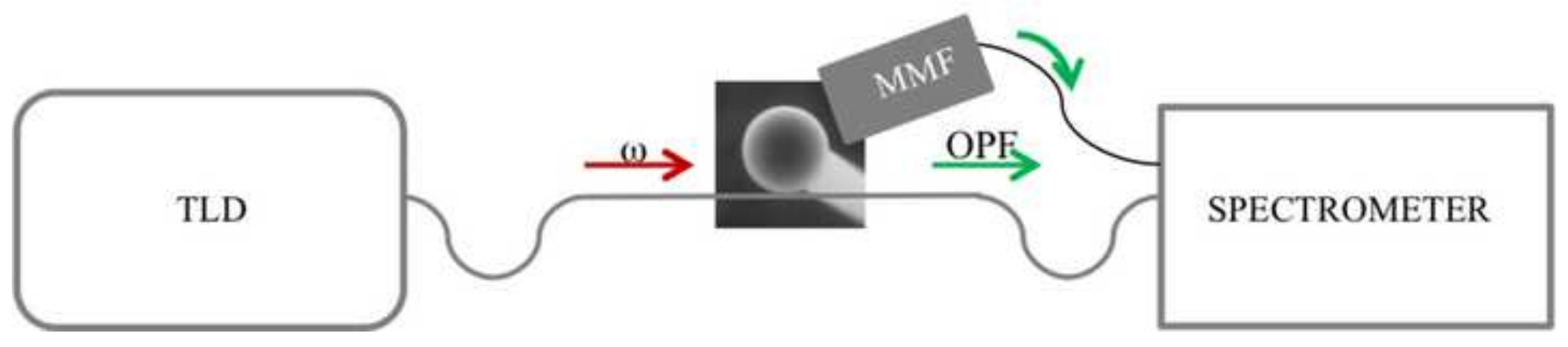

\section{Click here to download high resolution image}

TLD

促

.
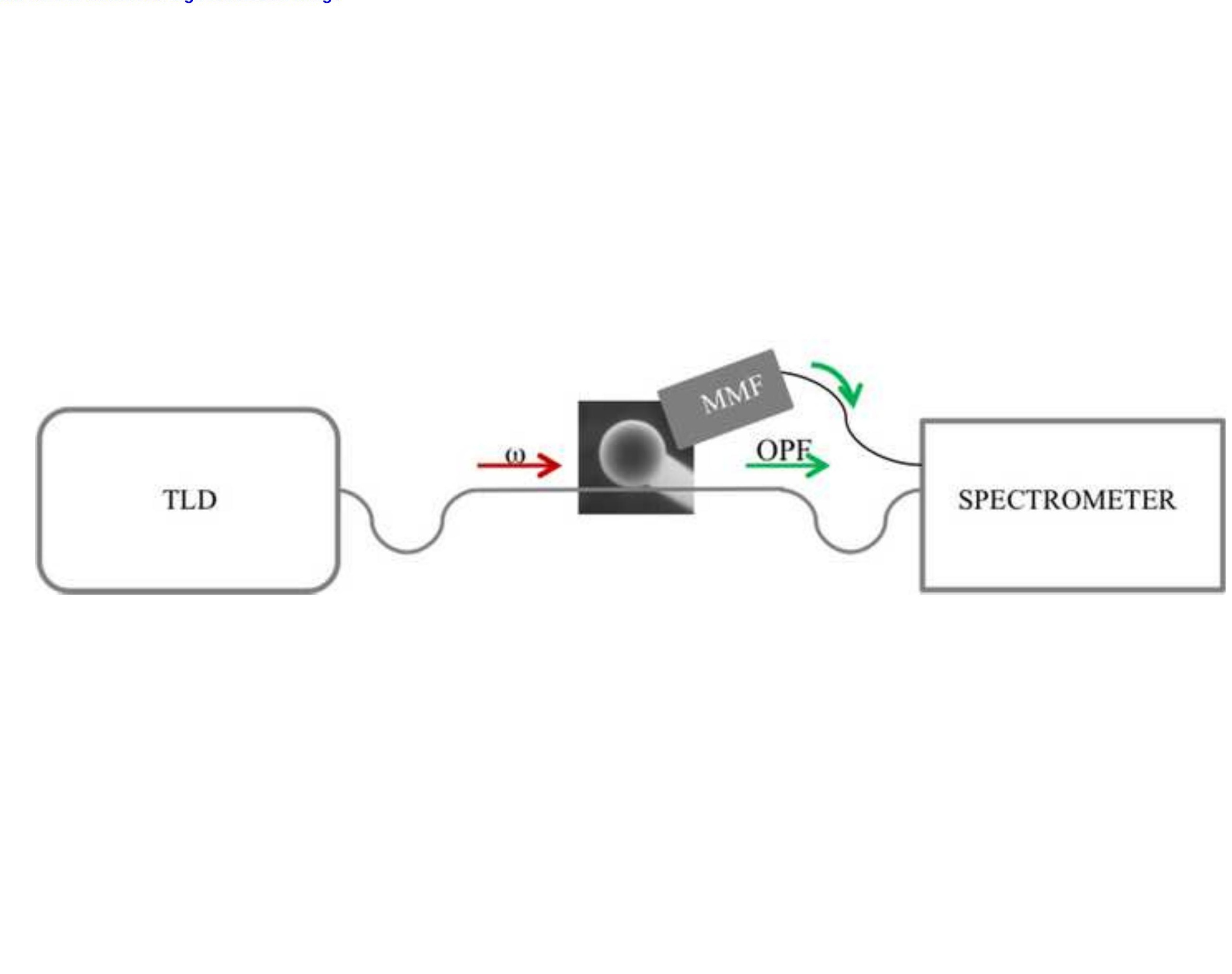

SPECTROMETER

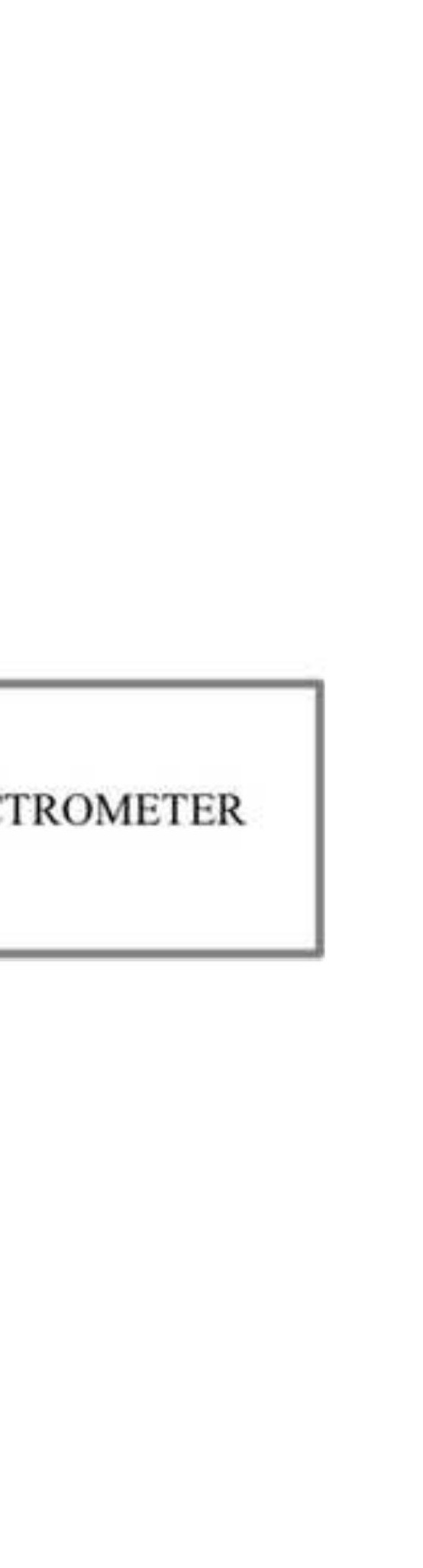




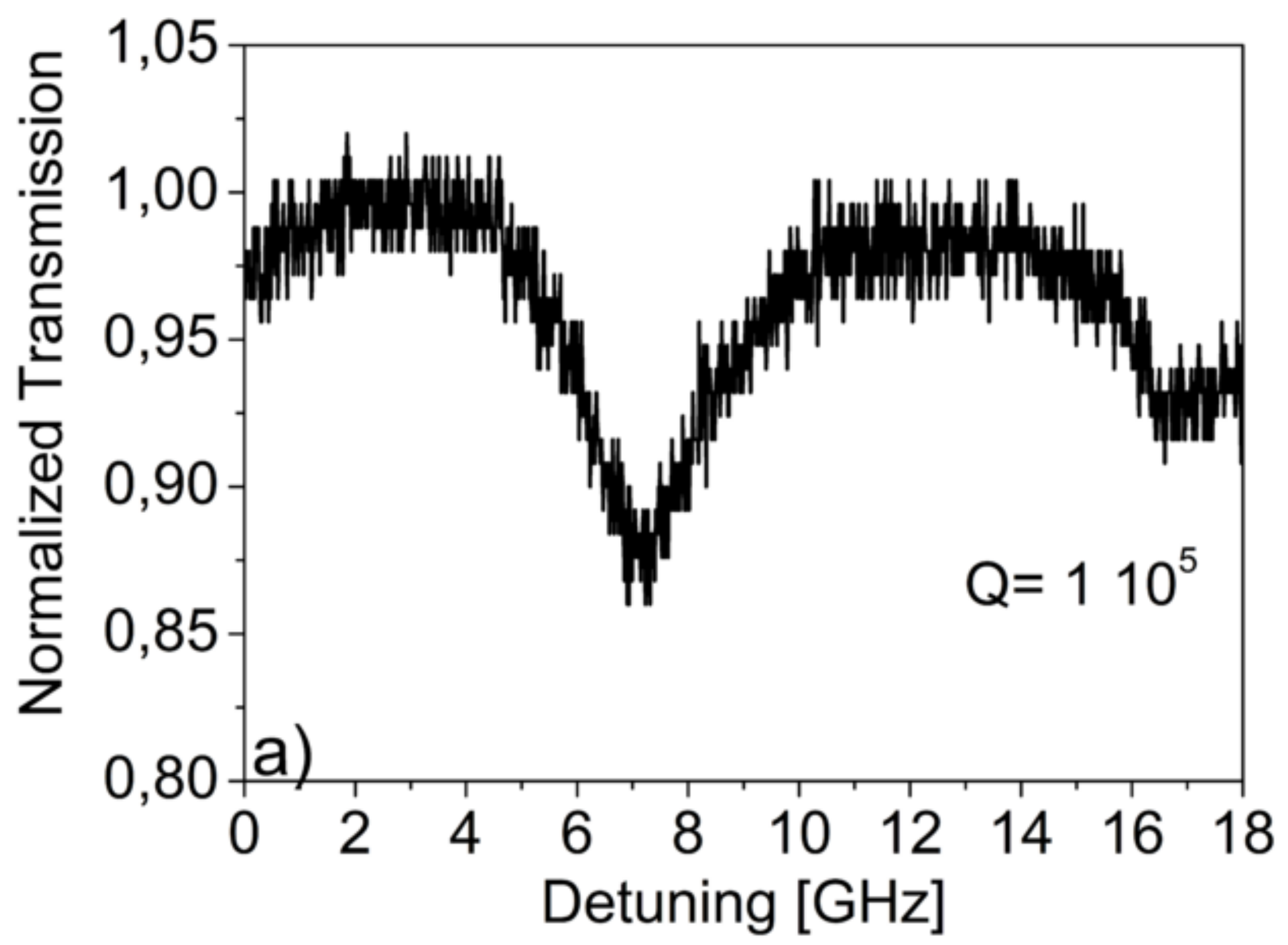




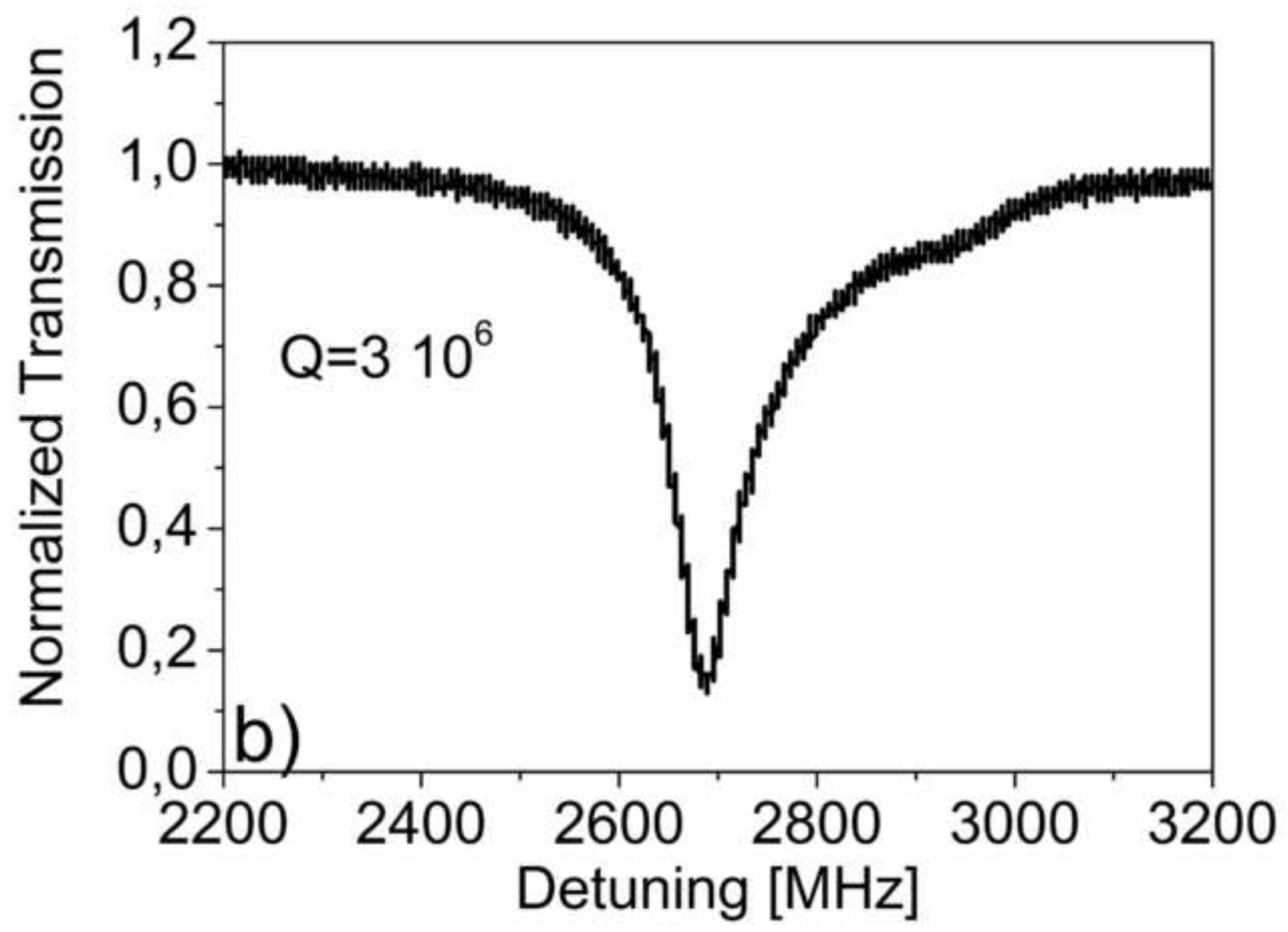




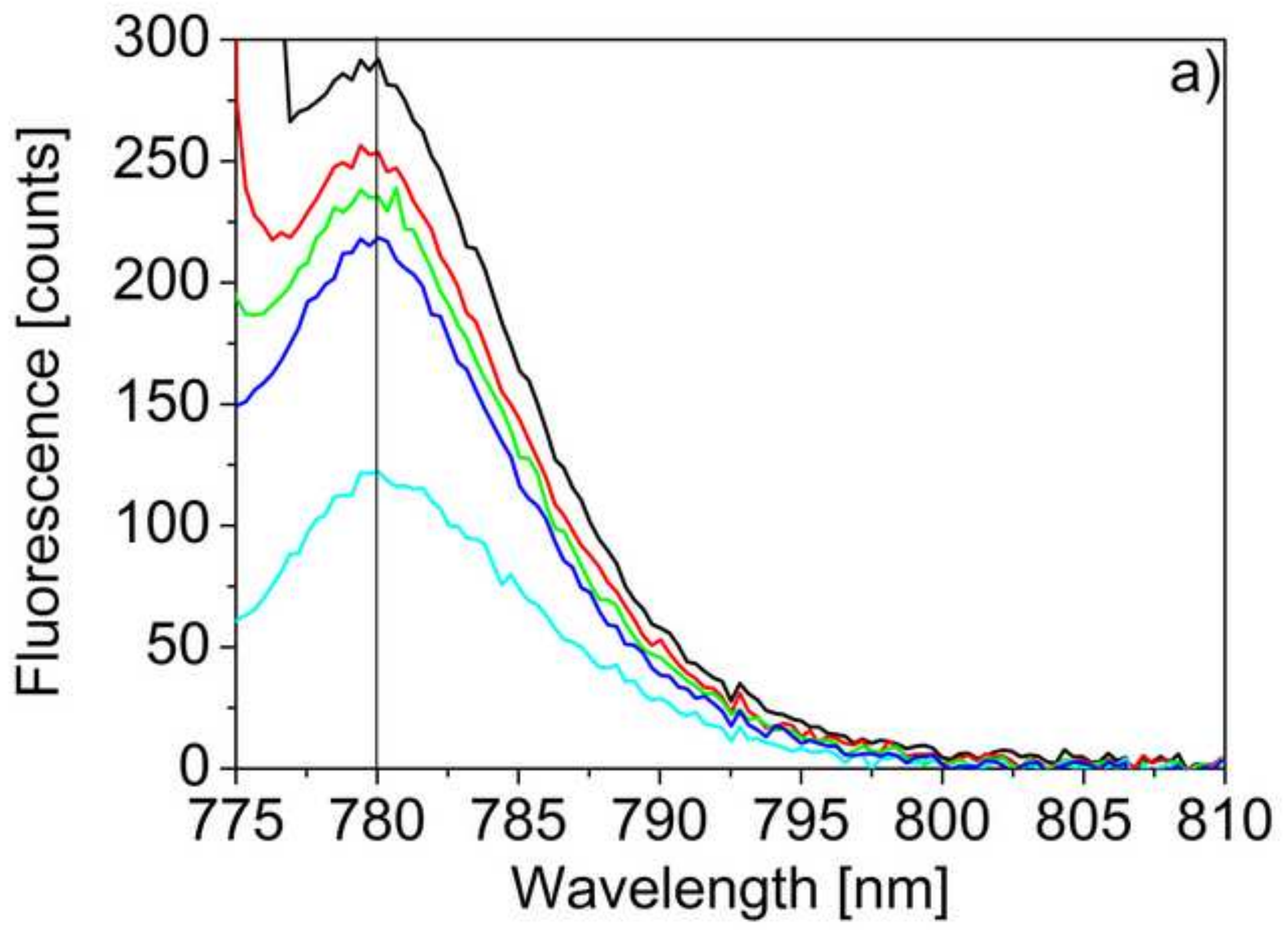




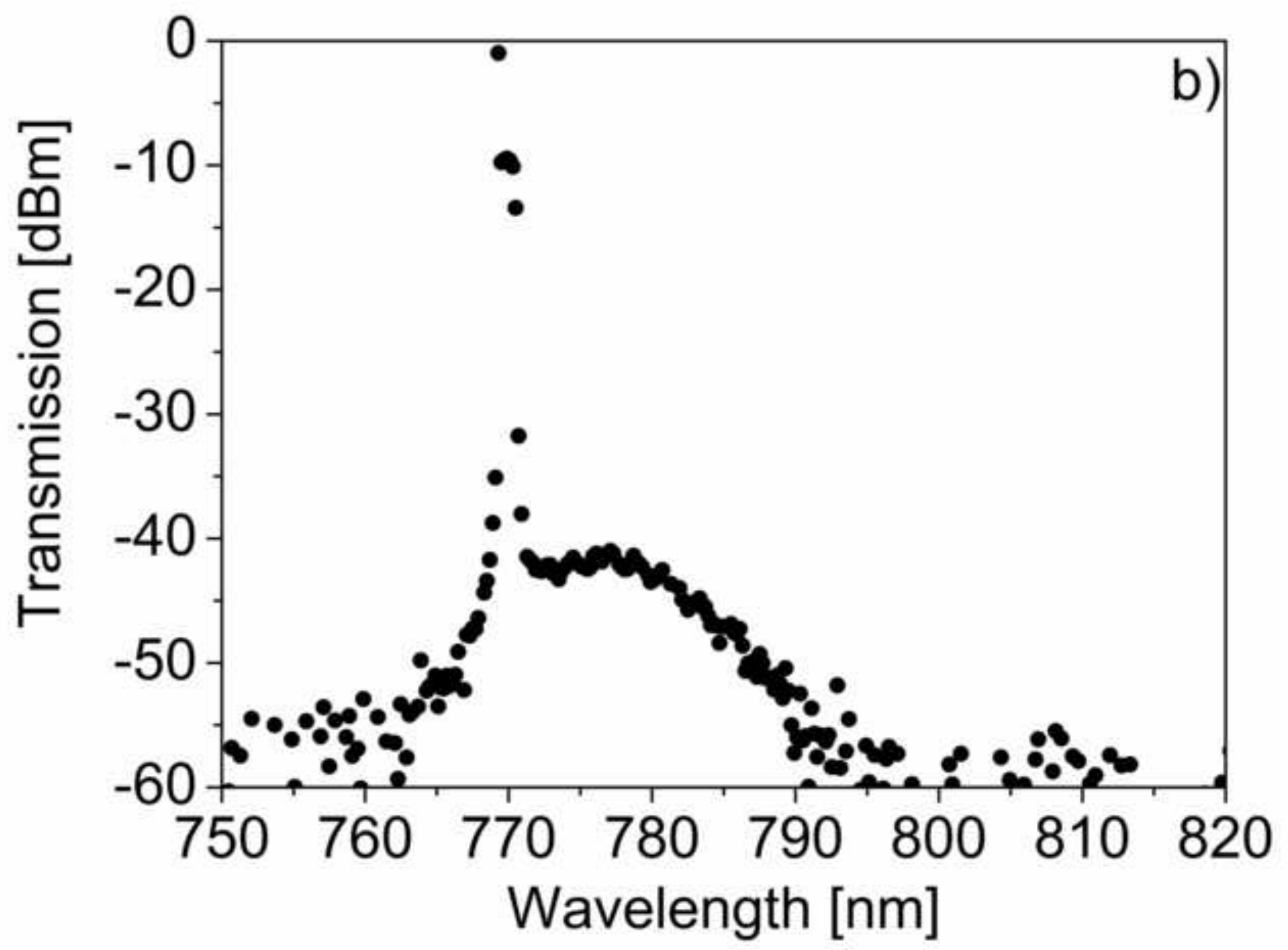




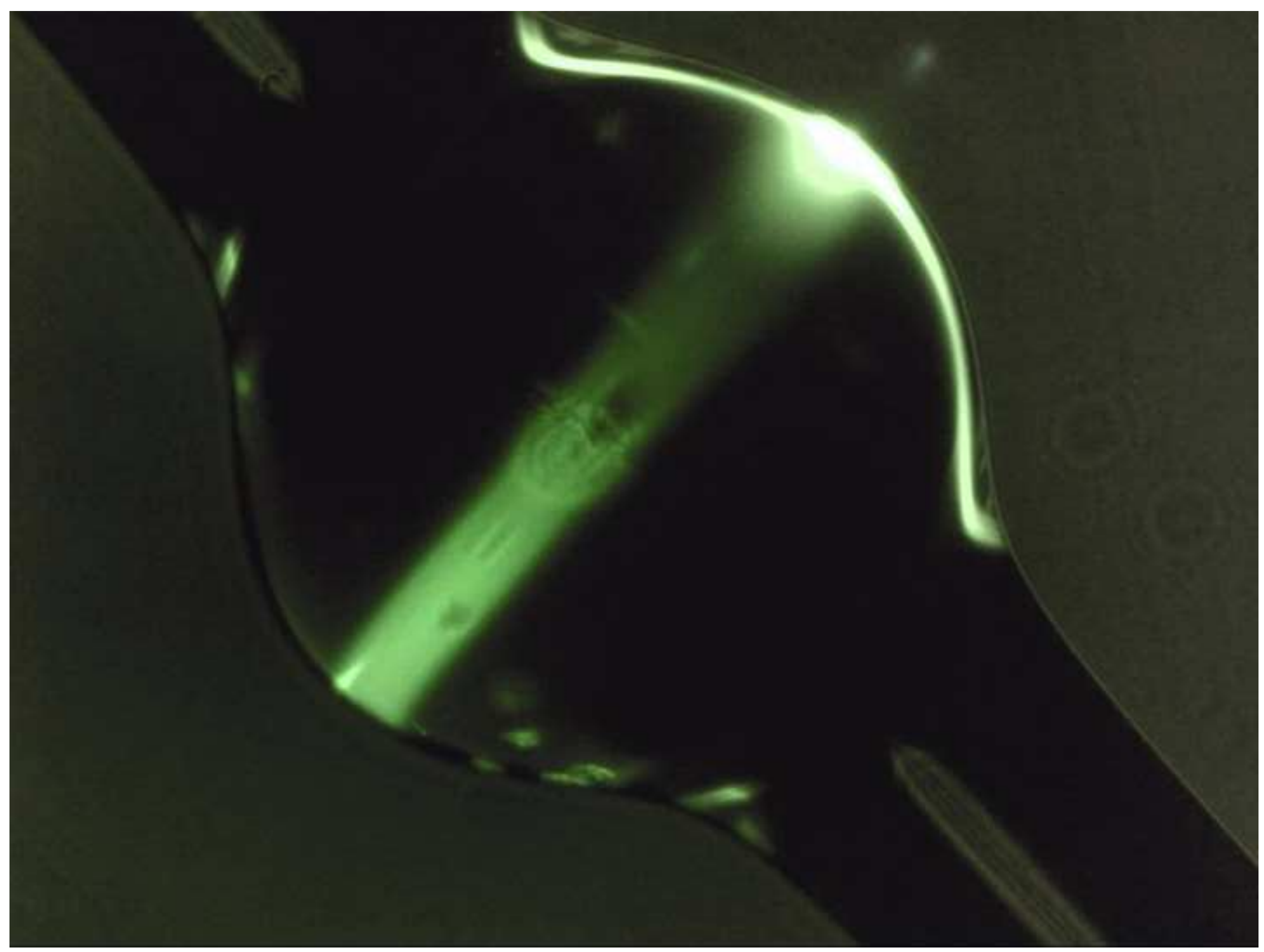

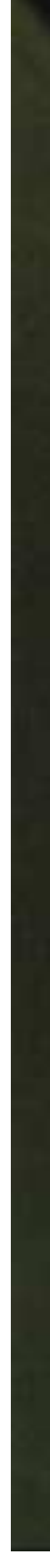




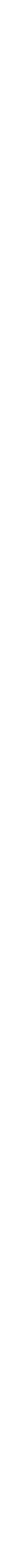




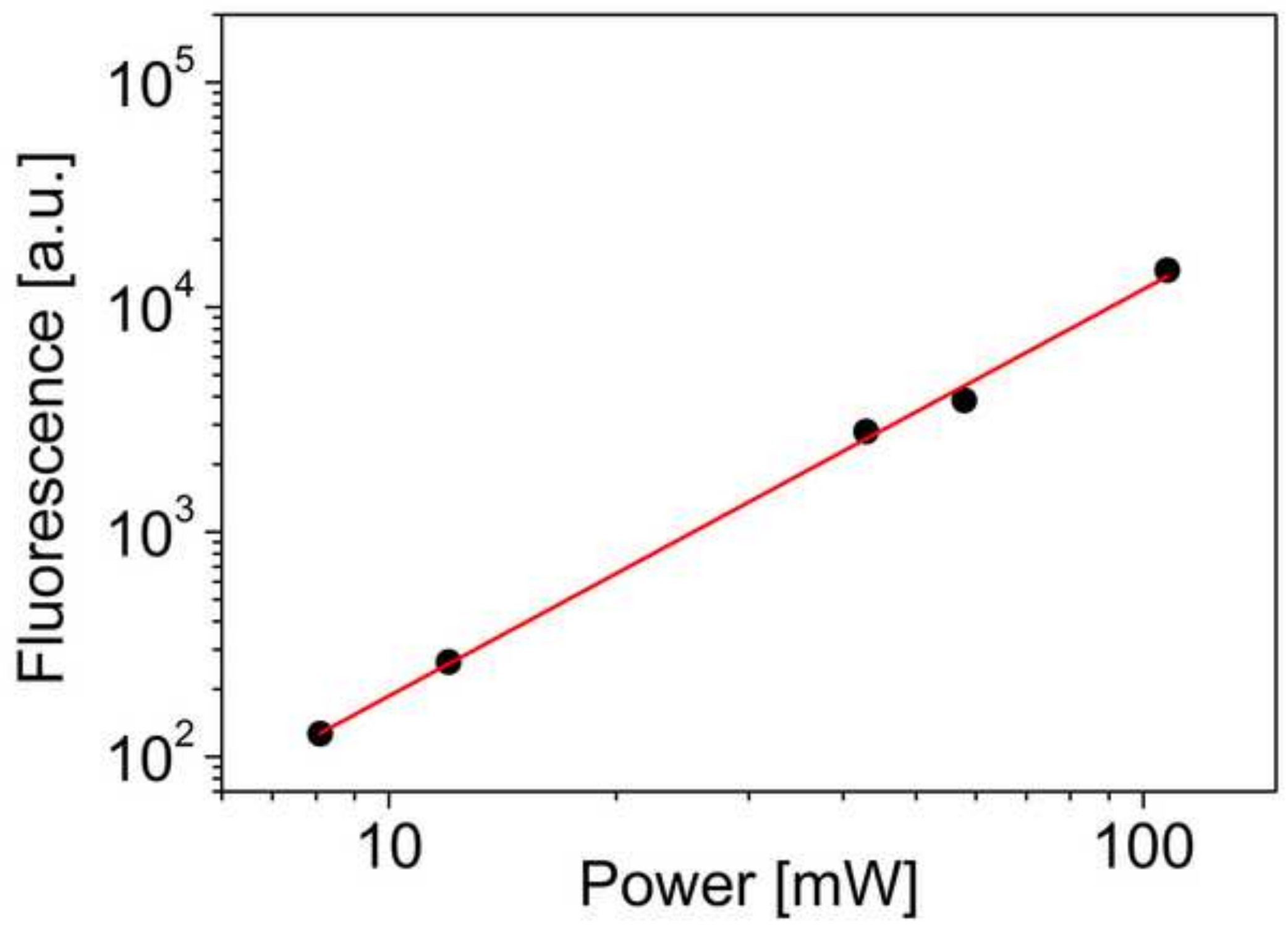


Click here to download high resolution image

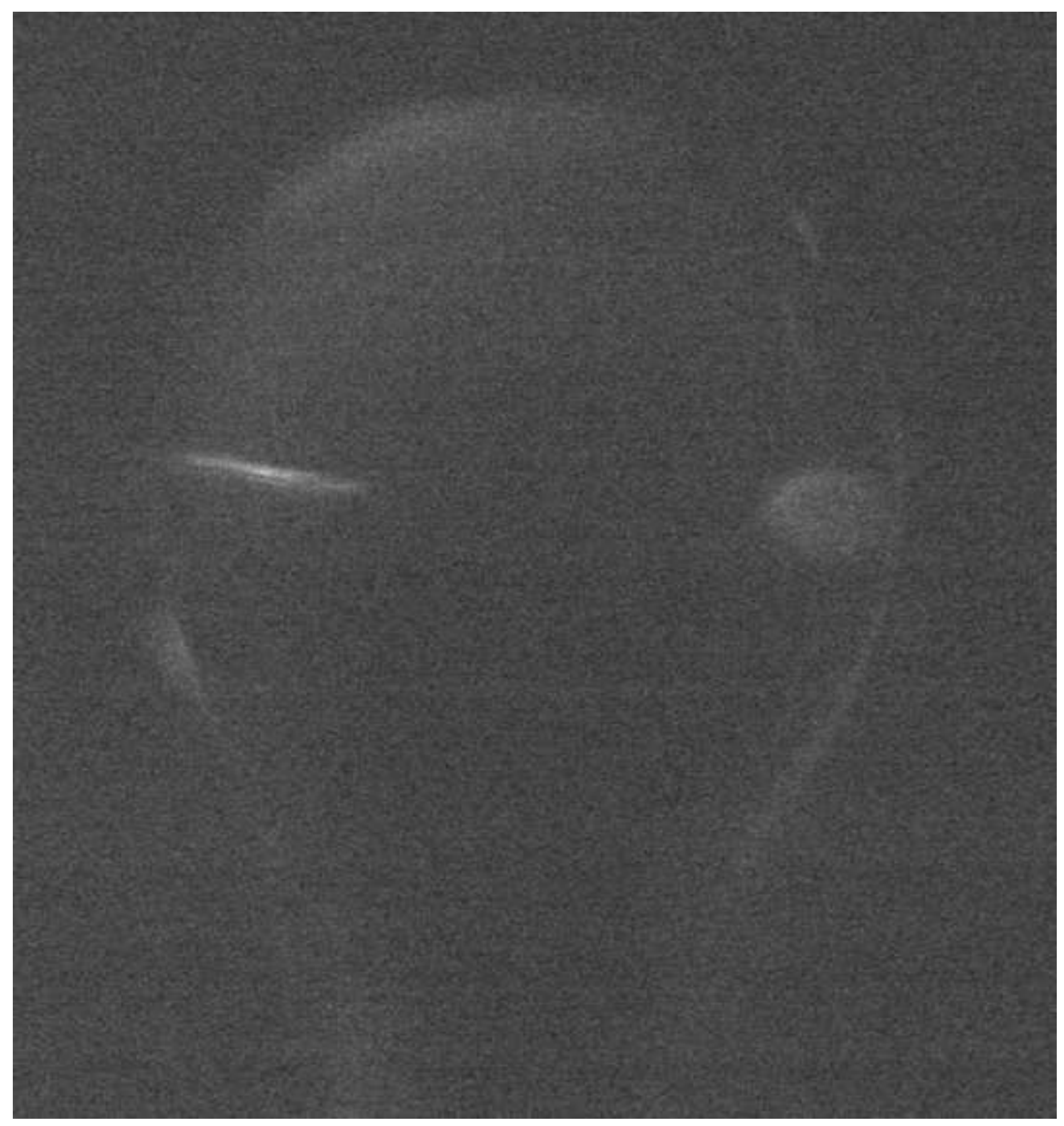

